\title{
Studying the interaction between gyrase and DNA using magnetic tweezers
}

\author{
ZHANG ZhiQiang, LIU YuRu, XIE Ping, LI Wei, DOU ShuoXing \& WANG PengYe* \\ Key Laboratory of Soft Matter Physics, Beijing National Laborartory for Condensed Matter Physics, Institute of Physics, Chinese Academy of \\ Sciences, Beijing 100190, China
}

Received April 9, 2012; accepted May 20, 2012

\begin{abstract}
The DNA gyrase of Escherichia coli plays an essential role in the life of this microorganism. It is unique among all topoisomerases because of its ability to introduce negative supercoils into DNA. This study investigated the single molecular interaction of $E$. coli gyrase with DNA using magnetic tweezers. The results showed that, in the absence of ATP, gyrase weakly binds the G and T segments. The stretched force of $0.7 \mathrm{pN}$ can gradually destroy the binding, whereas that of $5.9 \mathrm{pN}$ directly destroys it. Addition of high concentrations of norfloxacin enhances gyrase binding to both segments, making them adapt to $5.9 \mathrm{pN}$. DNA gyrase reduces the plectonemic dimension, which was determined by the bacterial enzyme and not by the pull force. Moreover, it has different affinities for positive supercoils, which it prefers, and negative supercoils. The time distribution of the dissociation of gyrase from DNA has a double-exponential form. We herein propose a model to explain this distribution and compare the results with those of other models.
\end{abstract}

gyrase, DNA, supercoil, magnetic tweezers

Citation: Zhang Z Q, Liu Y R, Xie P, et al. Studying the interaction between gyrase and DNA using magnetic tweezers. Chin Sci Bull, 2012, 57: 3560-3566, doi: $10.1007 / \mathrm{s} 11434-012-5420-8$

Topoisomerases are important and conserved enzymes extensively found in the biosphere. They adjust the topological structure of DNA by introducing and removing supercoils, knots, and catenations, which are correlated with fundamental and crucial DNA processes, including replication, transcription, repair, and reorganization [1-3].

Topoisomerases can be divided into two categories: type I topoisomerase and type II topoisomerase. Type I topoisomerase temporarily cuts one strand of double-stranded DNA, rotating two strands to remove the negative and positive DNA supercoils. Type II topoisomerase temporarily cuts one DNA segment (G segment) of double-stranded DNA and catalyzes another segment ( $\mathrm{T}$ segment) for passage, thus introducing or removing the supercoiled structure. Each cycle of type II topoisomerase is able to change the DNA ligase by 2 [4-7]. Gyrase is a type II topoisomerase that is composed of two subunits, namely, GyrA and GyrB,

*Corresponding author (email: pywang@aphy.iphy.ac.cn) which form a $\mathrm{A}_{2} \mathrm{~B}_{2}$ heterotetramer [3]. DNA gyrase is the only known topoisomerase that can introduce negative supercoils into DNA [8]. It removes the activity of knots and catenations at low levels [9-11], and it is crucial for bacteria. The fact that gyrase does not exist in the human body renders it an excellent antibiotic target. Quinolone is a widely applied antibiotic that targets gyrase [12].

Numerous approaches have been applied to studying the interaction between gyrase and DNA. Digestion experiments of DNA enzyme I foot printing and exonuclease III have shown that the segment combined with DNA gyrase is approximately $140 \mathrm{bp}$ [13-15]. This segment winds around the C-terminal domain of the GyrA subunit via the righthanded supercoil $[16,17]$. Its crystal structure [18-20] reveals that the integration of gyrase and DNA will generate extensive rearrangement of the configuration of gyrase. Meanwhile, the G segment is intensively twisted by gyrase. Most studies on this topic have focused on observing the static interaction between gyrase and DNA. In contrast, the 
present study dynamically observed the real-time binding and dissociation of gyrase and DNA using a single-molecule manipulation device.

\section{Materials and methods}

\subsection{DNA structuring and experimental setup}

The DNA used in this experiment was composed of three parts [21], a $1 \mathrm{~kb}$ segment located at two ends and multiply marked with biotin and digoxigenin as well as a middle segment with two length specifications (10 and $1.5 \mathrm{~kb}$ ). These segments are amplified from the $\lambda$ phage DNA through polymerase chain reaction. Magnetic tweezers, which have been detailed described [22], were used in the present study. In brief, one end of DNA was connected to a superparamagnetic bead marked with streptavidin (Dynal Company) with a $1 \mu \mathrm{m}$ diameter, whereas the other end was connected to the glass surface covered with anti-digoxigenin. Two NdFeB magnets were placed above the sample. When the magnets were moved close to the sample, pull force could be applied to DNA through the superparamagnetic bead. Meanwhile, torque was applied to DNA by rotating the magnet. The position of the bead was recorded using an inverted microscope at the sampling frequency of $60 \mathrm{~Hz}$ (Figure 1).

\subsection{Reaction conditions}

All reactions between Escherichia coli gyrase (NEB Company) and DNA were carried out at $25^{\circ} \mathrm{C}$. The buffer was composed of $35 \mathrm{mmol} / \mathrm{L}$ Tris- $\mathrm{HCl}, 24 \mathrm{mmol} / \mathrm{L} \mathrm{KCl}$, $4 \mathrm{mmol} / \mathrm{L} \mathrm{MgCl}_{2}, 2 \mathrm{mmol} / \mathrm{L} \mathrm{DTT}, 5 \mathrm{mmol} / \mathrm{L}$ spermidine, $0.1 \mathrm{mg} / \mathrm{mL}$ of BSA and $6.5 \%$ glycerol.

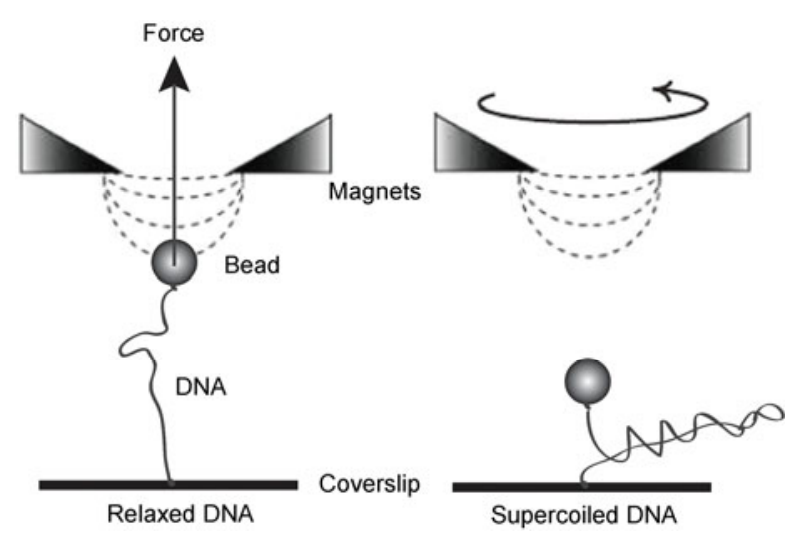

Figure 1 Diagram of a magnetic tweezers. One end of DNA is connected to the magnetic DNA bead, and the other end is connected to the coverslip. Pull force could be applied to DNA through the superparamagnetic bead, whereas torque is applied to DNA by rotating the magnet. When the number of turns exceeds the threshold, a supercoiled structure of DNA will be formed to shorten the DNA extension.

\section{Results}

\subsection{The binding of topoisomerases to the $\mathbf{G}$ and $T$ segments}

In this study, one end of the $10 \mathrm{~kb}$ DNA molecule (linking number $L k_{0}=935$ ) was linked to the coverslip, whereas the other end was connected to a superparamagnetic bead with a diameter of $1 \mu \mathrm{m}$. When the magnet is rotated, it triggers the bead to rotate. The rotating bead introduces torque into the DNA, provided the DNA strands have no nick. When the torque exceeds the threshold, plectonemic structures of DNA will be formed. Magnetic tweezers record the change of DNA extension. In the process, the supercoiling density can be obtained according to the following equation: $\sigma \equiv$ $\Delta L k / L k_{0}=n / L k_{0}$, where $L k_{0}$ is the linking number of relaxed DNA, $\Delta L k$ is the change of linking number and $n$ is the rotation numbers. In our magnetic tweezers equipment, $\Delta L k$ equals to $n$ because the rotation of beads tightly couples the rotation of magnets.

We first rotated the magnet to introduce positive supercoils into the DNA molecule $(\sigma=0.05)$ and then stretched the DNA using small $(F=0.7 \mathrm{pN})$ and strong $(F=5.9 \mathrm{pN})$ forces alternately to observe the change in DNA length. In the absence of gyrase, when stretching force rapidly increasing (the time required $<0.1 \mathrm{~s}$ ), the plectonemic structures of DNA was pulled open such that DNA length increased in a fast, smooth, and continuous manner (data not shown), consist with previous studies [23,24].

The above-described experiment was repeated with the addition of $E$. coli gyrase $(0.43 \mathrm{nM})$. Positively supercoiled DNA $(\sigma=0.06)$ was stretched using small $(F=0.06 \mathrm{pN})$ and strong $(F=0.7 \mathrm{pN})$ forces alternately. When $0.7 \mathrm{pN}$ was applied, the DNA length initially increased rapidly, but then slowly and gradually increased to the maximum (Figure 2(a)). The DNA length which slowly and gradually increased was approximately $500 \mathrm{~nm}$ (Figure 2(a)) (the dimension of a plectoneme is about $22.9 \mathrm{~nm}$, see next section), indicating that $E$. coli gyrase simultaneously binds the $G$ segment and the $\mathrm{T}$ segment. Several plectonemic structures can thus be temporarily clamped and stabilized. Moreover, these data demonstrated that the binding between gyrase and DNA molecule is relatively weak and will be gradually pulled open under $0.7 \mathrm{pN}$ of force.

The strong force in the above-described experiments was increased to $5.9 \mathrm{pN}$ to further elucidate the binding of gyrase and DNA. The forces of $0.06 \mathrm{pN}$ and $5.9 \mathrm{pN}$ were alternately applied to stretch DNA with $\sigma=0.03$. When the force was increased, the plectonemic structures of DNA were rapidly destroyed. Approximate $62 \%$ of 69 events, DNA molecules reached the maximum value directly. The remaining 38\% demonstrated a temporary pause (lasting less than $10 \mathrm{~s}$ ) close to the maximum (Figure 2(b)), indicating weak binding between gyrase and DNA. In contrast to DNA gyrase, eukaryotic topo II strongly binds with DNA. It 

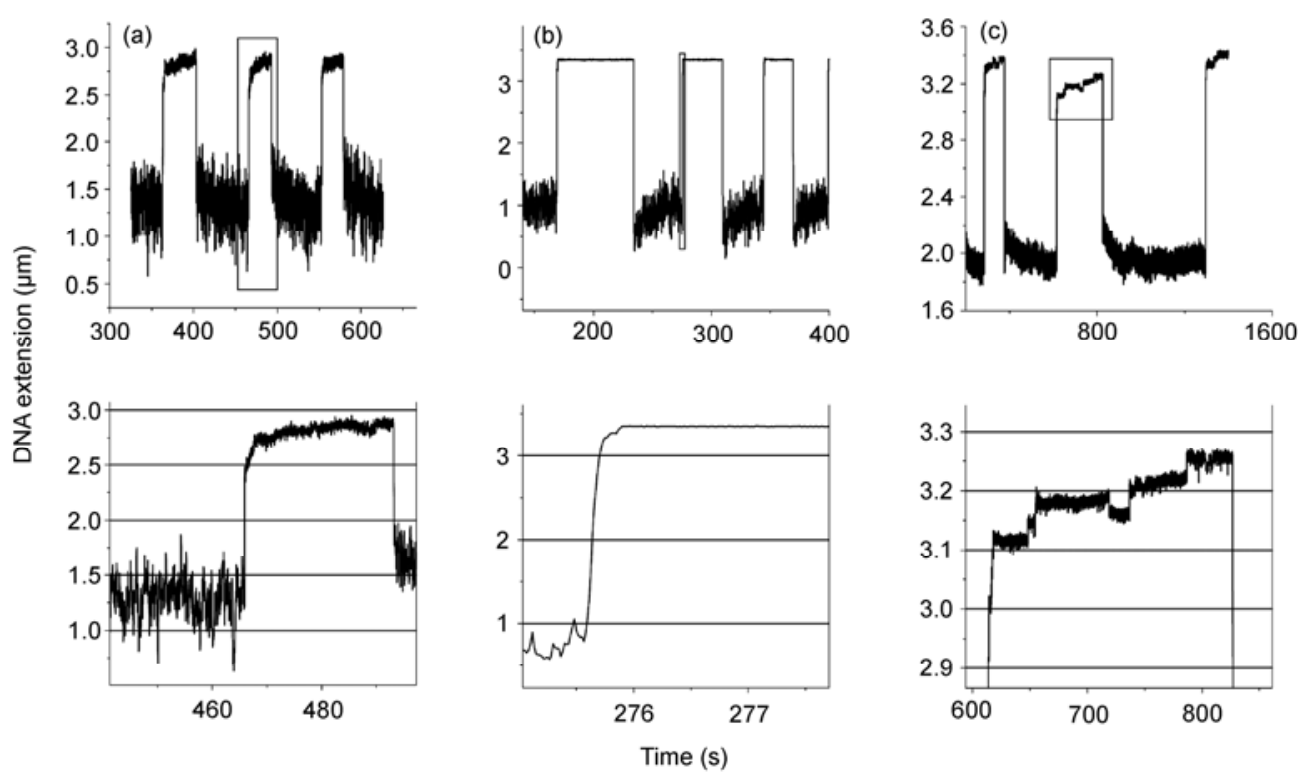

Figure 2 Changes in DNA length under different pull forces after the binding of gyrase $(0.43 \mathrm{nM})$ and DNA (10 $\mathrm{kb})$. (a) The pull force alternated between $0.06 \mathrm{pN}$ and $0.7 \mathrm{pN}$, and DNA was in the positively supercoiled state $(\sigma=0.06)$. When strong force was applied, DNA length at first increased rapidly and then increased slowly to the maximum value. (b) The force alternated between $0.06 \mathrm{pN}$ and $5.9 \mathrm{pN}$, and DNA was in the positively supercoiled state $(\sigma=$ $0.03)$. When strong force was applied, DNA length increased to the maximum value at a faster rate. (c) Norfloxacin $(30 \mu \mathrm{M})$ was added, and the force alternated between $0.7 \mathrm{pN}$ and $5.9 \mathrm{pN}$ when DNA was in the positively supercoiled state $(\sigma=0.05)$. When strong force was applied, DNA length increased rapidly to $3.1 \mu \mathrm{m}$. Then plateaus occurred (lasting longer than $50 \mathrm{~s}$ ), and DNA length slowly increased to the maximum. The hopping part of the length is enlarged to clearly display the details of length variation. The bottom row represents enlarged images of the frame shown in the upper row.

can maintain its binding with the $\mathrm{G}$ and $\mathrm{T}$ segments when 5 $\mathrm{pN}$ of force is applied [24]. To this end, the above-described experiment was carried out with topo II. When the force was increased, the length did not reach the maximum value directly and the plateau phase lasted for a long period (after $40 \mathrm{~s}, 89 \%$ did not release).

Norfloxacin at $30 \mu \mathrm{M}$ was subsequently added. The forces of $0.7 \mathrm{pN}$ and $5.9 \mathrm{pN}$ were applied alternately to stretch DNA with $\sigma=0.05$. When the force was increased, the length rapidly increased to a certain value, a plateau phase occurred (lasting longer than $50 \mathrm{~s}$ ), and the length increased gradually. These results suggest that norfloxacin enhanced the binding between gyrase and DNA. In order to verify the phenomenon caused by the action of both gyrase and DNA, control experiments were performed. In presence of norfloxacin but in absent of gyrase, we repeated above experiments. The results are similar with those of both are absent. Thus, it implied that the phenomenon shown Figure 2(c) is the results of gyrase and norfloxacin simultaneously action.

\subsection{The binding of gyrase on supercoiled DNA}

We next studied the impact of gyrase on equilibrium length and supercoiling density $\sigma=n / L k_{0}$ of DNA when the constant tension of $0.15 \mathrm{pN}$ was applied. A sufficient number of studies have been conducted on the response of DNA length to the supercoil in the absence of gyrase [25,26]. Under forces $<0.4 \mathrm{pN}$, when the number of magnet turns exceeded a certain threshold $n_{\mathrm{c}}$, DNA length started to buckle to form plectonemes. The changes in length induced by negative and positive supercoils are symmetrical. The relation curve between DNA length and supercoiling density was of a typical bell shape, and the maximum value appeared at $\sigma=0$ (Figure 3). When gyrase was added, the length changes introduced by positive and negative supercoiling remained symmetrical; however, the modified length for each turn became smaller, i.e. gyrase reduced the geometric scale of the supercoiled structure of DNA.

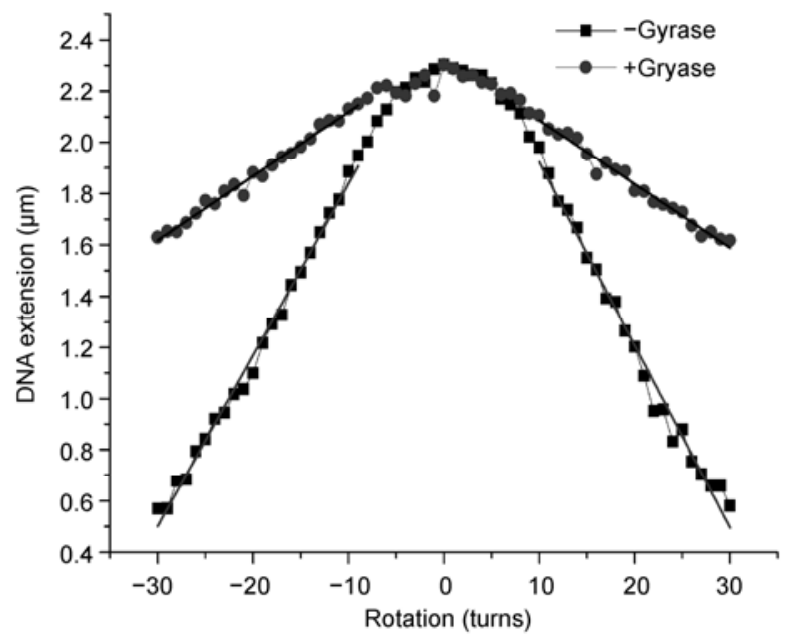

Figure 3 The constant tension of $0.15 \mathrm{pN}$ was applied to DNA. Square dots represent values without DNA gyrase, whereas circular dots represent those with $0.43 \mathrm{nM}$ DNA gyrase. The relation curve between DNA length and number of magnet turns is also shown. In the supercoiled area, the relation curve between DNA length and number of turns can be fitted (with the thick line) satisfactorily. The slope of these lines was calculated. 
As shown in Figure 3, in the supercoiled area, the slope of the lines was calculated. In the absence of DNA gyrase and under $0.15 \mathrm{pN}$, for each turn of the magnet, the length was reduced $\delta l_{0}=75.2 \pm 5.6 \mathrm{~nm}$. When $0.43 \mathrm{nM}$ gyrase was added, the reduced length became smaller and differed for the positive and negative supercoils. For the positive supercoil, the length was reduced $\delta l_{\mathrm{p}}^{+}=22.9 \pm 4.2 \mathrm{~nm}$; for the negative supercoil, the length was reduced $\delta l_{\mathrm{p}}{ }^{-}=28.0 \pm 7.0$ $\mathrm{nm}$. These results indicate that when $F$ was $<1.0 \mathrm{pN}$ (Figure 4(a) and (b)), gyrase improved DNA to produce tighter supercoils.

We also calculated the slope of DNA length variations for the positive and negative supercoils using different gyrase concentrations (Figure 4(c)). For the positive supercoil, $0.05 \mathrm{nM}$ gyrase obviously reduced the slope. The slope decreased with increasing gyrase concentration. For the negative supercoil, $0.2 \mathrm{nM}$ gyrase concentrations still did not affect the slope. Therefore, chiral differences in the binding between gyrase and DNA were detected.

In the absence of protein in the solution, $\delta l_{0}$ clearly changed with the changing pull force. In the presence of DNA gyrase, $\delta l_{\mathrm{p}}{ }^{+}$and $\delta l_{\mathrm{p}}^{-}$did not have significant effects with the changing pull force (Figure 4(a) and (b)). These re- sults indicate that upon the addition of gyrase, the scale of the supercoiled structure no longer depended on the pull force by DNA but on the interaction between gyrase and DNA.

\subsection{Dynamics of the binding and unbinding at buck- ling threshold}

We used the short DNA molecule of $1.5 \mathrm{~kb}$, which can effectively improve the signal-to-noise ratio, to study the binding and dissociation kinetics of gyrase and DNA molecule. During the experiment, DNA was stretched under the force of $2.9 \mathrm{pN}$ and the buckling threshold $n_{\mathrm{c}}=5$. The magnet was rotated 5 turns to the threshold value. Because free energy of forming plectonemes or not are equivalent, DNA shifts between the two states. The formation of a plectoneme will shorten the DNA, and magnetic tweezers can clearly observe the corresponding length changes in DNA (data not shown but are reported in [27,28]). After gyrase was added, the time which DNA remained in the supercoiled state significantly extended (Figure 5(a), II), because the binding of gyrase made the DNA supercoils more stable. However, the binding of DNA and gyrase was reversible and they would dissociate after the gyrase-DNA
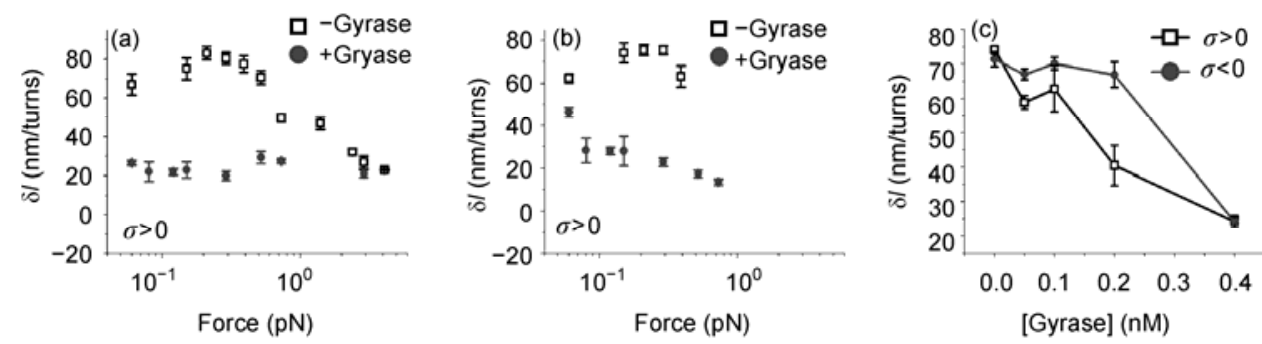

Figure 4 (a) The reduced length of positively supercoiled DNA $\delta l$ for each turn was used as a function of the applied force with or without 0.43 nM gyrase. (b) The same experiment was conducted for negatively supercoiled DNA. When the force was greater than 0.4 pN, the negatively supercoiled DNA melted. The error bar represents standard deviation. (c) Relation curve between the slope of length changes in DNA and the concentration of gyrase.
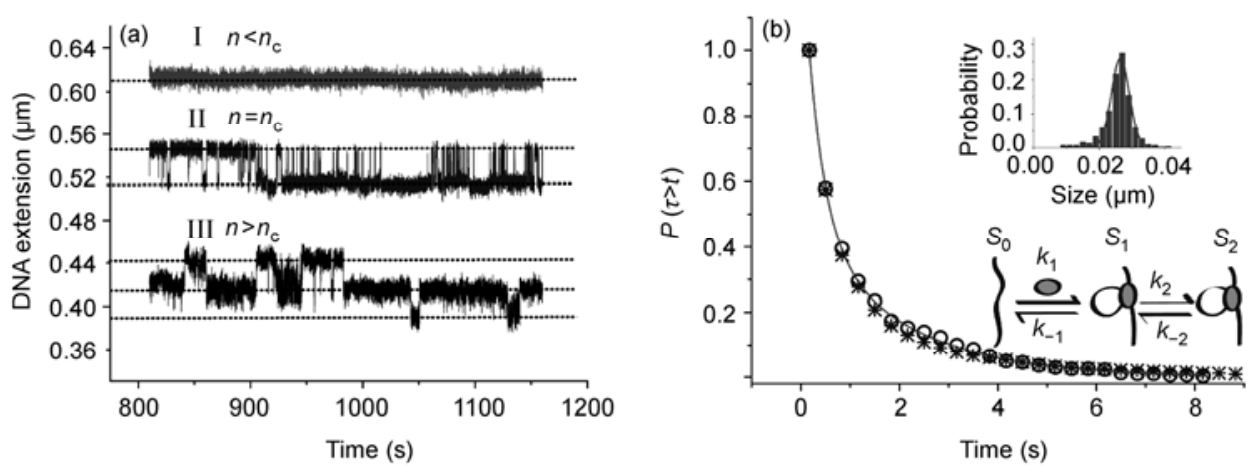

Figure 5 Dynamics of the binding of DNA gyrase with $1.5 \mathrm{~kb}$ DNA at $2.9 \mathrm{pN}$ force. (a) With gyrase added, DNA length changed with time. I, II, and III correspond to DNA length when the number of turns $n$ comparing the buckling threshold $n_{\mathrm{c}}$ was $n=2<n_{\mathrm{c}}, n=5=n_{\mathrm{c}}$, and $n=6>n_{\mathrm{c}}$, respectively. The figure was processed with vertical translation to avoid overlapping. We extracted the length changes and binding time. The dotted line represents the average length of corresponding DNA. (b) When $n=n_{\mathrm{c}}$, the cumulative probability distribution of the time that DNA gyrase dissociated from DNA (black circle, 299 bindings). The star points represent the distribution obtained from our model. The double-exponential distribution (line) fits the experimental results satisfactorily. The duration of $66 \%$ of the binding lasted $0.34 \mathrm{~s}$, whereas that of the remaining $34 \%$ was $1.96 \mathrm{~s}$. The illustration reflects the distribution histogram of the length change of DNA. According to Gaussian fitting, the average value was $26.4 \mathrm{~nm}$ and the standard deviation was $5.5 \mathrm{~nm}$. The sketch map represents the two-state model of DNA gyrase binding to DNA. Based on the cumulative probability distribution, the kinetic constants $k_{-1}, k_{2}$, and $k_{-2}$ could be obtained. 
complex had existed for a while, thus DNA supercoils relaxed and DNA extension increased. After that, DNA spontaneously formed supercoils again, and then the above process repeated. When $n=2<n_{\mathrm{c}}$, length change in DNA was not observed even in the presence of gyrase (Figure 5(a), I), indicating that without ATP, at the protein concentration applied in the experiment, gyrase can bind on DNA supercoils but cannot bind on DNA without supercoils.

With further rotation of the small magnet to $n=6>n_{\mathrm{c}}$, several separate DNA lengths with an interval of nearly 25 $\mathrm{nm}$ can be observed (Figure 5(a), III). This value $(25 \mathrm{~nm})$ is very close to the value of the supercoiled structure of DNA under the force of $2.9 \mathrm{pN}$, indicating that each supercoil has bound with a DNA gyrase molecule. The separated DNA length corresponds to the binding and dissociation of several enzymes.

The distribution of time for the gyrase-DNA complex in the binding state was calculated at the threshold. Figure 5(b) shows the cumulative probability histogram of the binding state $P(\tau>t)(N=299)$ and that the distribution can be satisfactorily fitted by a double-exponential function: the duration of $66 \%$ of the binding $\left(t_{1}\right)$ was $0.34 \mathrm{~s}$, and that of the remaining $34 \%\left(t_{2}\right)$ was $1.96 \mathrm{~s}$. Given the single-exponential distribution with $t_{1}$, the possibility for values to be higher than $t_{2}$ was less than 2 among 299 events. However, 48 of these 299 events were detected with values greater than $t_{2}$, thereby suggesting that the distribution was unlikely to be a single-exponential one.

Similar results were observed for the binding of Drosophila topo II [24] and E. coli topo IV [23] with DNA. The researchers presented that the two exponential distributions indicate that the gyrase-DNA complex has two binding states with different strengths, and they proposed a corresponding kinetic model. The present study used their model to analyze the experimental data.

The state of DNA without gyrase was marked as $S_{0}$, weak binding of the gyrase-DNA complex was marked as $S_{1}$, and strong binding was marked as $S_{2} . k_{1}, k_{-1}, k_{2}$, and $k_{-2}$ represent kinetic parameters (Figure $5(\mathrm{~b})$ ). $P_{0}(t), P_{1}(t)$, and $P_{2}(t)$ represent the probabilities in $S_{0}, S_{1}$, and $S_{2}$ at $t$, respectively. The initial conditions $P_{0}(0)=P_{2}(0)=0$ and $P_{1}(0)=1$ were given. As it is of rare chance the same gyrase which dissociated from DNA to bind to the same position of DNA, $k_{1}=0$. The probability distribution $P$ for $\tau$ larger than $t$ is $P(\tau>t)=1-P_{0}(t)$.

The following ordinary differential equations [23] were solved:

$$
\begin{aligned}
\frac{\mathrm{d} P_{0}}{\mathrm{~d} t} & =k_{-1} P_{1}, \\
\frac{\mathrm{d} P_{1}}{\mathrm{~d} t} & =-\left(k_{-1}+k_{2}\right) P_{1}+k_{-2} P_{2}, \\
\frac{\mathrm{d} P_{2}}{\mathrm{~d} t} & =k_{2} P_{1}-k_{-2} P_{2} .
\end{aligned}
$$

Yields

$$
P(\tau>t)=\frac{k_{-1}+\lambda_{-}}{\lambda_{-}-\lambda_{+}} \mathrm{e}^{\lambda_{+} t}-\frac{\lambda_{+}+k_{-1}}{\lambda_{-}-\lambda_{+}} \mathrm{e}^{\lambda_{-} t}
$$

with

$$
\lambda_{ \pm}=-\frac{1}{2}\left(k_{-1}+k_{2}+k_{-2} \pm \sqrt{\left(k_{-1}+k_{2}+k_{-2}\right)^{2}-4 k_{-1} k_{-2}}\right) \text {. }
$$

The experimental data on the cumulative probability distribution $P(\tau>t)$ can be fitted satisfactorily with the above functions (Figure 5), yielding the kinetic constants of binding and dissociation.

\subsection{Another explanation for the dynamics}

Another explanation similar to the interpretation of the pause of RNA polymerase and $\lambda$ exonuclease [29,30] is herein proposed for the double-exponential time distribution of gyrase dissociation. The free energy of the binding of gyrase and the $\mathrm{G}$ and $\mathrm{T}$ segments is calculated as $E$ and the interactional potential energy can be written in Morse code: $V(x)=E[\exp (-2 x / A)-2 \exp (-x / A)]$, where $2 A=1 \mathrm{~nm}($ Debye length) and the direction of $x$ is as explained in the legend to Figure 6. The dissociation of gyrase from the DNA molecule can be described by the Langevin equation:

$$
\gamma \frac{\mathrm{d} x}{\mathrm{~d} t}=-\frac{\mathrm{d} V(x)}{\mathrm{d} x}+\zeta(t)
$$

where the viscous load acting on gyrase is contained in the drag coefficient $\gamma=6 \pi \eta R_{\mathrm{g}}=9.4 \times 10^{-11} \mathrm{~kg} \mathrm{~s}^{-1}$, with $\eta=0.01$ $\mathrm{g} \mathrm{cm}^{-1} \mathrm{~s}^{-1}$ and gyrase being considered as a sphere of radius $R_{\mathrm{g}}=5 \mathrm{~nm}$, and $\zeta(t)$ is the thermal noise, with $\langle\zeta(t)\rangle=0$ and $\left\langle\zeta(t) \zeta\left(t^{\prime}\right)\right\rangle=2 k_{B} T \gamma \delta\left(t-t^{\prime}\right)$. The time distribution of gyrase dissociation can be obtained using eq. (4), which has a single-exponential form: $f(t)=\exp (-t / T) / T$.

Given the differences in DNA sequence, the binding energies $E$ of the $\mathrm{G}$ and $\mathrm{T}$ segments and gyrase showed subtle

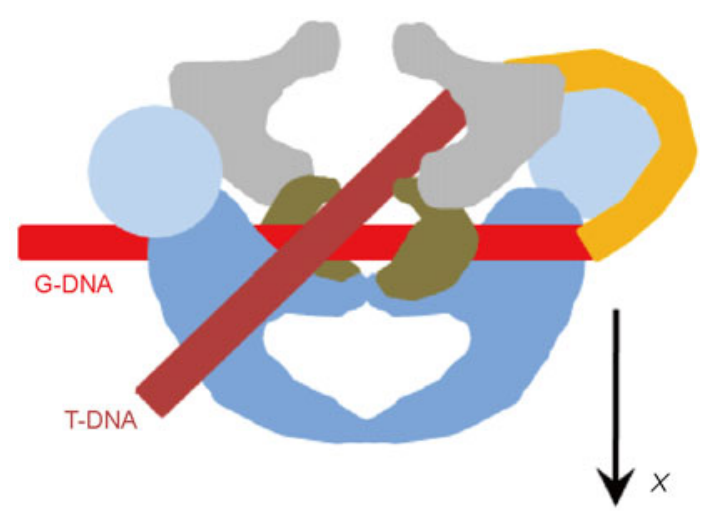

Figure 6 Structure of the gyrase-DNA complex. $x$ indicates the direction of the dissociation of gyrase from the $\mathrm{G}$ and $\mathrm{T}$ segments. 
differences and distinct single-exponential distributions. To this end, the time distribution of the dissociation of gyrase from several supercoiled DNA molecules is no longer of a single-exponential form but in the form of the linear sum of several exponential distributions, as

$$
\sum_{i=1}^{N}\left[C_{i} \cdot \exp \left(-t / T_{i}\right)\right],
$$

where $N$ is an integer larger than or equal to 2. The cumulative probability distribution of dissociation time also takes this form. This distribution can be satisfactorily fitted by the linear sum of two exponential distributions. The star points in Figure 5(b) represent the cumulative probability distribution of dissociation time simulated by our model. Here, we assumed four binding energies: $E=17.3 k_{\mathrm{B}} T(T \approx 0.2 \mathrm{~s}$, accounting for $17.1 \%), E=18 k_{\mathrm{B}} T(T \approx 0.41 \mathrm{~s}$, accounting for $37.4 \%), E=18.9 k_{\mathrm{B}} T(T \approx 0.9 \mathrm{~s}$, accounting for $11.4 \%)$, and $E=19.7 k_{\mathrm{B}} T(T \approx 1.74 \mathrm{~s}$, accounting for $34.1 \%)$. The simulation results are consistent with the experimental results, indicating that even if only one binding state exists in the gyrase-DNA complex, due to the subtle differences of the binding energy $E$, the double-exponential distribution of dissociation time can still be generated.

In the absence of nucleotide, experimental results cannot demonstrate two configurations of the interaction between gyrase (topo II and topo IV) and DNA. Subtle and inevitable differences in the binding of gyrase with various DNA segments can be attributed to differences in DNA sequences. Such differences were verified in another experiment [31]. Thus, we propose that the dissociation time probability distribution of the double-exponential form is generated from differences of the binding energy between gyrase and different DNA segments.

\section{Discussion}

This study used a single-molecule manipulation based on magnetic tweezers to systematically and quantitatively evaluate the interaction between $E$. coli gyrase and DNA. Gyrase was found to bind both the $\mathrm{G}$ segment and the $\mathrm{T}$ segment of DNA with a relatively weak binding. The presence of norfloxacin made the binding of gyrase and DNA more stable. According with our results, biochemistry research [32] indicated that, norfloxacin almost did not bind with gyrase or DNA when only one of them existed; but a large number of norfloxacin was bound if both existed. Moreover, biochemistry research [33] and crystal structure [34] showed that norfloxacin together with DNA and gyrase formed a stable complex where norfloxacin bound near active site of GyrA Tyr122 and interact with GyrB and DNA. Besides, gyrase could change the geometric scale of DNA supercoils, yielding chiral differences, clearly indicating that it has different effects on positive and negative super- coils. Dissociation time of gyrase from DNA is consistent with a double-exponential distribution.

Studies on crystal structures [18-20] have shown that a substantial rearrangement of the $\mathrm{G}$ segment is generated from binding with gyrase. This segment has $140 \mathrm{bp}$ intertwined at the C-terminal end of the GyrA subunit, which explains why the geometric scale of the supercoiled structure is reduced after the addition of DNA gyrase and why the dimension is determined by gyrase rather than by the pull force applied on DNA.

The elucidation of interactions of DNA gyrase and DNA absent from ATP is helpful to understand the working mechanism of DNA gyrase and facilitate understanding and interpretation of single-molecule experimental data on DNA gyrase activity after the addition of ATP. DNA gyrase imposed different effects on positive and negative supercoils and could more easily bind with positively supercoiled DNA. These findings are conducive to our understanding of the different activities of gyrase for positive and negative supercoils.

The binding between bacterial gyrase and DNA is similar to that involving topo IV, but rather different from eukaryote topo II. Without ATP, the binding between gyrase and the $\mathrm{G}$ and $\mathrm{T}$ segments is relatively weak and reversible, whereas that between topo II and the two segments is strong and irreversible. This difference stems from the structural differences between gyrase and topo II, with the crystal structure of DNA gyrase [18-20] showing that the C-terminal domain of GyrA can intensively bend DNA and twine DNA to the right. This segment is not found in eukaryotic topo II. Despite these, gyrase and topo II are similar in that their dissociation time from DNA are consistent with a double-exponential distribution and can be interpreted by the model proposed herein.

This work was supported by the National Natural Science Foundation of China $(10834014,10974248)$ and the National Basic Research Program of China (2009CB930704).

1 Charvin G, Strick T R, Bensimon D, et al. Tracking topoisomerase activity at the single-molecule level. Ann Rev Biophys Biomol Struct, 2005, 34: 201-219

2 Schoeffler A J, Berger J M. DNA topoisomerases: Harnessing and constraining energy to govern chromosome topology. Q Rev Biophys, 2008, 41: 41-101

3 Champoux J J. DNA topoisomerases: Structure, function, and mechanism. Ann Rev Biochem, 2001, 70: 369-413

4 Brown P O, Cozzarelli N R. A sign inversion mechanism for enzymatic supercoiling of DNA. Science, 1979, 206: 1081-1083

5 Liu L F, Rowe T C, Yang L, et al. Cleavage of DNA by mammalian DNA topoisomerase II. J Biol Chem, 1983, 258: 15365-15370

6 Xie P. Dynamics of strand passage catalyzed by topoisomerase II. Eur Biophys J, 2010, 39: 1251-1259

7 Morrison A, Cozzarelli N R. Site-specific cleavage of DNA by $E$. coli DNA gyrase. Cell, 1979, 17: 175-184

8 Gellert M, Mizuuchi K, O’Dea M H, et al. DNA gyrase: An enzyme that introduces superhelical turns into DNA. Proc Natl Acad Sci USA, 1976, 73: 3872-3876 
9 Zechiedrich E L, Cozzarelli N R. Roles of topoisomerase IV and DNA gyrase in DNA unlinking during replication in Escherichia coli. Genes Dev, 1995, 9: 2859-2869

10 Liu L F, Liu C C, Alberts B M. Type II DNA topoisomerases: Enzymes that can unknot a topologically knotted DNA molecule via a reversible double-strand break. Cell, 1980, 19: 697-707

11 Kreuzer K N, Cozzarelli N R. Formation and resolution of DNA catenanes by DNA gyrase. Cell, 1980, 20: 245-254

12 Drlica K. Mechanism of fluoroquinolone action. Curr Opin Microbiol, 1999, 2: 504-508

13 Fisher L M, Mizuuchi K, O’Dea M H, et al. Site-specific interaction of DNA gyrase with DNA. Proc Natl Acad Sci USA, 1981, 78: 4165-4169

14 Kirkegaard K, Wang J C. Mapping the topography of DNA wrapped around gyrase by nucleolytic and chemical probing of complexes of unique DNA sequences. Cell, 1981, 23: 721-729

15 Morrison A, Cozzarelli N R. Contacts between DNA gyrase and its binding site on DNA: Features of symmetry and asymmetry revealed by protection from nucleases. Proc Natl Acad Sci USA, 1981, 78: 1416-1420

16 Ruthenburg A J, Graybosch D M, Huetsch J C, et al. A superhelical spiral in the Escherichia coli DNA gyrase A C-terminal domain imparts unidirectional supercoiling bias. J Biol Chem, 2005, 280: 26177-26184

17 Liu L F, Wang J C. DNA-DNA gyrase complex: The wrapping of the DNA duplex outside the enzyme. Cell, 1978, 15: 979-984

18 Dong K C, Berger J M. Structural basis for gate-DNA recognition and bending by type IIA topoisomerases. Nature, 2007, 450: 12011205

19 Costenaro L, Grossmann J G, Ebel C, et al. Small-angle X-ray scattering reveals the solution structure of the full-length DNA gyrase a subunit. Structure, 2005, 13: 287-296

20 Corbett K D, Shultzaberger R K, Berger J M. The C-terminal domain of DNA gyrase A adopts a DNA-bending beta-pinwheel fold. Proc Natl Acad Sci USA, 2004, 101: 7293-7298

21 Revyakin A, Ebright R H, Strick T R. Single-molecule DNA nanomanipulation: improved resolution through use of shorter DNA fragments. Nat Methods, 2005, 2: 127-138

22 Gosse C, Croquette V. Magnetic tweezers: Micromanipulation and force measurement at the molecular level. Biophys J, 2002, 82: 3314-3329

23 Charvin G. Topoisomerase IV bends and overtwists DNA upon binding. Biophys J, 2005, 89: 384-392

24 Strick T R, Croquette V, Bensimon D. Single-molecule analysis of DNA uncoiling by a type II topoisomerase. Nature, 2000, 404: 901904

25 Clauvelin N, Audoly B, Neukirch S. Elasticity and electrostatics of plectonemic DNA. Biophys J, 2009, 96: 3716-3723

26 Strick T R, Allemand J F, Bensimon D, et al. Behavior of supercoiled DNA. Biophys J, 1998, 74: 2016-2028

27 Brutzer H, Luzzietti N, Klaue D, et al. Energetics at the DNA supercoiling transition. Biophys J, 2010, 98: 1267-1276

28 Daniels B, Forth S, Sheinin M, et al. Discontinuities at the DNA supercoiling transition. Phys Rev E, 2009, 80: 040901

29 Xie P. A dynamic model for transcription elongation and sequencedependent short pauses by RNA polymerase. Biosystems, 2008, 93: 199-210

30 Xie P. Molecular motors that digest their track to rectify Brownian motion: Processive movement of exonuclease enzymes. J PhysCondens Mat, 2009, 21: 375108

31 Pato M L, Howe M M, Higgins N P. A DNA gyrase-binding site at the center of the bacteriophage $\mathrm{Mu}$ genome is required for efficient replicative transposition. Proc Natl Acad Sci USA, 1990, 87: 8716-8720

32 Shen L L, Kohlbrenner W E, Weigl D, et al. Mechanism of quinolone inhibition of DNA gyrase. Appearance of unique norfloxacin binding sites in enzyme-DNA complexes. J Biol Chem, 1989, 264: 29732978

33 Heddle J, Maxwell A. Quinolone-binding pocket of DNA gyrase: Role of GyrB. Antimicrobial Agents Chemotherapy, 2002, 46: 18051815

34 Laponogov I, Sohi M K, Veselkov D A, et al. Structural insight into the quinolone-DNA cleavage complex of type IIA topoisomerases. Nat Struct Mol Biol, 2009, 16: 667-669

Open Access This article is distributed under the terms of the Creative Commons Attribution License which permits any use, distribution, and reproduction in any medium, provided the original author(s) and source are credited. 